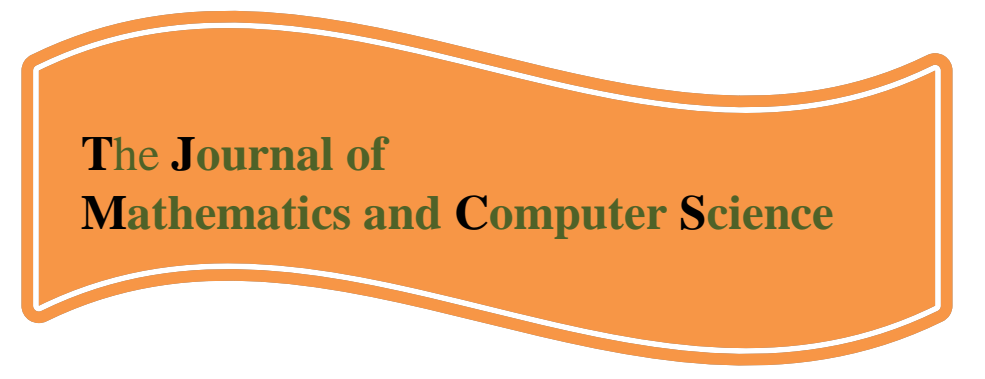

Available online at

http://www.TJMCS.com

The Journal of Mathematics and Computer Science Vol .5 No. 1 (2012) 22-27

\title{
Fixed Point and Hyers-Ulam-Rassias Stability of a Quadratic Functional Equation in Menger Probabilistic Normed Spaces
}

\author{
Ehsan Movahednia \\ Behbahan Khatam Al-Anbia University of Tecnology \\ ehsar.m@gmail.com \\ Sara Eshtehar \\ Behbahan Khatam Al-Anbia University of Tecnology \\ seshtehar@yahoo.com
}

Received: November 2012, Revised: December 2012

Online Publication: December 2012

\section{Abstract}

In this paper, using the fixed point alternative approach, we investigate the Hyers Ulam-Rassias stability of the quadratic functional equation

in Menger probabilistic normed spaces.

$$
f(x+y)+f(x-y)=2 f(x)+2 f(y)
$$

Keywords: fixed point theory, Hyers-Ulam-Rassias stability.

2010 Mathematics Subject Classification: Primary 54A40; Secondary 46 S40.

\section{Introduction.}

In [1] K. Menger proposed the probabilistic concept of distance by replacing the number $d(p, q)$, as distance between points $(p, q)$ by a distribution function $F_{(p, q)}$. This idea led to a large development of probabilistic analysis [1],[2].

Probabilistic normed spaces were first defined by Serstnev in [3]. So, a fruitful theory concordant with that of ordinary normed spaces and with that of probabilistic metric spaces was initiated. The theory of probabilistic normed spaces is important as a random generalization of deterministic 
linear normed space theory. in the same time it gives also new tools in the study of random operator equations. For important results of probabilistic functional analysis we refer to [1],[2],[4].

A classical question in the theory of functional equations is the following: When is it true that a function which approximately satisfies a functional equation must be close to an exact solution of the equation? If the problem accepts a solution, we say that the equation is stable. The first stability problem concerning group homomorphisms was raised by Ulam [5] in 1940. In the next year D.H. Hyres [6], gave a positive answer to the above question for additive groups under the assumption that the groups are Banach spaces.

Subsequently the result of Hyers was generalized by Aoki [8] for additive mapping and by Th. M. Rassias [4] for linear mapping by considering an unbounded cauchy difference. The paper of Th. M. Rassias [4] has provided a lot of influence in the development of what we call the Hyers-Ulam stability or the Hyers-Ulam-Rassias stability of functional equations.

The functional equation

$$
f(x+y)+f(x-y)=2 f(x)+2 f(y)
$$

is called a quadratic functional equation. In particular, every solution of the quadratic functional equation is said to be a quadratic mapping. A generalized Hyers-Ulam stability problem for the quadratic functional equation was proved by Skof [8] for mappings $f: X \longrightarrow Y$ where $X$ is a normed space and $Y$ is a Banach space.

In 1996, G. Isac and Th. M. Rassias [6] were the first to provide applications of stability theory of functional equations for the proof of new fixed point theorems with applications. Some authors considered the stability of quadratic functional equation random normed space [22] . By fixed point methods, the stability problems of several functional equations have been extensively investigated by a number of authors(see [9],[10],[11]).

Definition 1.1. A function $f: R \rightarrow[0,1]$ is called adistribution function if it is nondecreasing and left-continuous, with $\sup _{t \in R} F(t)=1$ and inf ${ }_{t \in R} F(t)=0$.

The class of all distribution functions $F$ with $F(0)=0$ is denoted by $D_{+} \cdot \varepsilon_{0}$ is the element of $D_{+}$ define by

$$
\varepsilon_{0}= \begin{cases}1 & t>0 \\ 0 & t \leq 0\end{cases}
$$

Definition 1.2. A binary operation $*:[0,1] \times[0,1] \rightarrow[0,1]$ is said to be a t-norm if it satisfies the following condition:

(1) * is commutative and associative;

(2) * is continuous;

(3) $a * 1=a$ for all $a \in[0,1]$;

(4) $a^{*} b \leq c^{*} d$ whenever $a \leq c$ and $b \leq d$ for all $a, b, c, d \in[0,1]$.

Definition1.3. [13] Let $X$ be a real vector space, $F$ a mapping from $X$ to $D_{+}$(for any $x \in X$, $F(X)$, is denoted by $\left.F_{x}\right)$ and ${ }^{*}$ a t-norm. The triple $\left(X, F,{ }^{*}\right)$ is called a Menger probabilistic normd spaces (briefly Menger PN-space) if the following conditions are satisfied:

(1) $F_{x}(0)=0$, for all $x \in X$;

(2) $F_{x}(0)=\varepsilon_{0}$ iff $x=\theta$;

(3) $F_{\alpha x}(t)=F_{x}\left(\frac{t}{|\alpha|}\right)$ for all $\alpha \in R, \alpha \neq 0$ and $x \in X$;

(4) $F_{x+y}\left(t_{1}+t_{2}\right) \geq F_{x}\left(t_{1}\right) * F_{y}\left(t_{2}\right)$ for all $x, y \in X$ and $t_{1}, t_{2}>0$.

Definition 1.4. Let $(X, F, *)$ be a Menger PN-space and let $\left\{x_{n}\right\}$ be a sequence in $X$. Then $\left\{x_{n}\right\}$ is said to be convergent if there exists $x \in X$ such that 


$$
\lim _{n \rightarrow \infty} F_{x_{n}-x}(t)=1
$$

For all $t>0$. In this case, $x$ is called the limit of $\left\{x_{n}\right\}$.

Definition 1.5. The sequence $\left\{x_{n}\right\}$ in Menger PN-space $(X, F, *)$ is called Cauchy if for each $\varepsilon>0$ and $\delta>0$ there exists some $n_{0}$ such that $F_{x_{n}-x_{m}}(\delta)>1-\varepsilon$ for all $m, n>n_{0}$.

Clearly, every convergent sequence in Menger PN-space is Cauchy. If each Cauchy sequence is convergent sequence in a Menger PN-space $(X, F, *)$, then $(X, F, *)$ is called Menger probabilistic Banach space (briefly, Menger PB-space).

Definition 1.6. Let $X$ be a set. A function $d: X \times X \rightarrow[0, \infty]$ is called a generalized metric on $X$ if satisfies the following conditions:

(1) $d(x, y)=0$ if and only if $x=y$ for all $x, y \in X$;

(2) $d(x, y)=d(y, x)$ for all $x, y \in X$;

(3) $d(x, z) \leq d(x, y)+d(y, z)$ for all $x, y, z \in X$;

Note that the only substantial difference of the generalized metric from the metric is that the range of generalized metric includes the infinity.

Theorem 1.1. Let (X,d) be a complete generalized metric space and $J: X \rightarrow X$ be a strictly contractive mapping with Lipschitz constant $L<1$. Then for all $x \in X$, either

$$
d\left(J^{n} x, J^{n+1} x\right)=\infty
$$

for all non-negative integers $n$ or there exists a positive integer $n_{0}$ such that

(1) $d\left(J^{n} x, J^{n+1} x\right)<\infty$ for all $n \geq n_{0}$;

(2) the sequence $\left\{J^{n} x\right\}$ converges to a fixed point $y^{*}$ of $J$;

(3) $y^{*}$ is the unique fixed point of $J$ in the set $Y=\left\{y \in X ; d\left(J^{n_{0}} x, y\right)<\infty\right\}$;

(4) $d\left(y, y^{*}\right) \leq \frac{1}{1-L} d(y, J y)$ for all $y \in Y$.

\section{Main results}

Throughout this section, using fixed point method, we prove the Hyers-Ulam-Rassias stability of quadratic functional equation in Menger probabilistic normed spaces.

Definition 2.1. Let $\left(X, F,{ }^{*}\right)$ be a Menger PN-spaces and $\left(Y, G,{ }^{*}\right)$ be a menger PB-spaces. A mapping $f: X \rightarrow Y$ is said to be P-approximately quadratic if

$$
G_{f(x+y)+f(x-y)-2 f(x)-2 f(y)}(t+s) \geq F_{x}(t) * F_{y}(s)
$$

for all $t, s>0$.

Theorem 2.1. Let $f: X \rightarrow Y$ be a P-approximately quadratic functional equation and there exists $0<\alpha<\frac{1}{4}$ such that

$$
F_{x}(2 t) \geq F_{\alpha x}(t)
$$

Then there exists a unique quadratic mapping $Q: X \rightarrow Y$ such that

$$
G_{f(x)-Q(x)}(t) \geq F_{x}\left(\frac{1-4 \alpha}{2 \alpha} t\right)
$$

Proof. Puttin $x=y$ and $s=t$ in (2.2), we have 


$$
G_{f(2 x)-4 f(x)}(2 t) \geq F_{x}(t)
$$

Replacing $x$ by $\frac{x}{2}$ in (2.5), we have

$$
G_{f(x)-4 f\left(\frac{x}{2}\right)}(2 t) \geq F_{\frac{x}{2}}(t)
$$

With the definition (1.3) and replacing $t$ by $\frac{t}{2}$ in (2.6) we obtain

$$
G_{f(x)-4 f\left(\frac{x}{2}\right)}(t) \geq F_{x}(t)
$$

for all $x \in X, \$ \mathrm{t}>0$. Consider the set $K:=\{g: X \rightarrow Y: g(0)=0\}$ and the generalized metric in $K$ defined by

$$
d(h, g)=\inf \left\{c \in[0, \infty] \mid G_{g(x)-h(x)}(c t) \geq F_{x}(t)\right\} .
$$

Where $\inf \varnothing=+\infty$. It is easy to show that $(K, d)$ is complete (see [22], lemma 2.1.). Now, we consider a linear mapping $J: K \rightarrow K$ such that $J h(x)=4 h\left(\frac{x}{2}\right)$ for all $x \in X$. First, we prove that $J$ is a strictly contractive mapping with the Lipschitz constant $4 \alpha$. In fact let $g, h \in K$ be such that $d(g, h)<c$. Then we have

$$
G_{g(x)-h(x)}(c t) \geq F_{x}(t)
$$

Whence

$$
\begin{aligned}
& G_{J g(x)-J h(x)}(4 \alpha c t)=G_{4 g\left(\frac{x}{2}\right)-4 h\left(\frac{x}{2}\right)}(4 \alpha c t) \\
&=G_{g\left(\frac{x}{2}\right)-h\left(\frac{x}{2}\right)}(\alpha c t) \\
& \geq F_{\frac{x}{2}}(\alpha t) \\
& \geq F_{x}(t)
\end{aligned}
$$

For all $x \in X, t>0$. Then

$$
d(J g, J h)<4 \alpha c
$$

This mean that

$$
d(J g, J h) \leq 4 \alpha d(g, h)
$$

for all $g, h \in K$. It follows from (2.7) that

$$
d(f, J f) \leq 2 \alpha
$$

Now, by Theorem (1.1) there exists a mapping $Q: X \rightarrow Y$ satisfying the following:

(1) $Q$ is a fixed point of $J$, that is ,

$$
Q\left(\frac{x}{2}\right)=\frac{1}{4} Q(x)
$$

for all $x \in X$. The mapping $Q$ is a unique fixed point of $J$ in the set

$$
\Omega=\{h \in K: d(g, h)<\infty\} .
$$

This implies that $Q$ is a unique mapping satisfying (2.13) such that there exists $c \in[0, \infty]$ satisfying

$$
G_{f(x)-Q(x)}(c t) \geq F_{x}(t)
$$

for all $x \in X$ and $t>0$. 
(2) $d\left(J^{n} f, Q\right) \rightarrow 0$ as $n \rightarrow \infty$. This implies the equality

$$
\lim _{n \rightarrow \infty} 4^{n} f\left(\frac{x}{2^{n}}\right)=Q(x)
$$

for all $x \in X$.

(3) $d(f, Q) \leq \frac{d(f, J f)}{1-L} \leq \frac{2 \alpha}{1-4 \alpha}$ with $f \in \Omega$ and so

$$
G_{f(x)-Q(x)}\left(\frac{2 \alpha t}{1-4 \alpha}\right) \geq F_{x}(t)
$$

This implies that

$$
G_{f(x)-Q(x)}(t) \geq F_{x}\left(\frac{1-4 \alpha}{2 \alpha}\right) .
$$

Then the inequality (2.4) holds. On the other hand

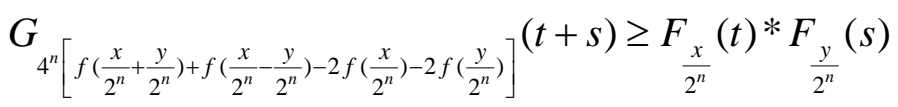

By definition (1.3) and (2.3) we have

$$
F_{\frac{x}{2^{n}}}(t) \geq F_{\alpha^{n} x}(t) \quad F_{\frac{y}{2^{n}}}(s) \geq F_{\alpha^{n} y}(s)
$$

So

$$
F_{\frac{x}{2^{n}}}(t) * F_{\frac{y}{2^{n}}}(s) \geq F_{\alpha^{n} x}(t) * F_{\alpha^{n} y}(s)
$$

for all $x, y \in X$ and $t, s>0$. Now, since $\lim _{n \rightarrow \infty} F_{\alpha^{n} x}(t)^{*} F_{\alpha^{n} y}(s)=1$ we have

$$
G_{Q(x+y)+Q(x-y)-2 Q(x)-2 Q(y)}(t+s)=1
$$

for all $x, y \in X$. This complete the proof.

\section{References.}

[1] K. Menger, Statistical metrics, Porceedings of the National Academy of Sciences of the United States of America, vol. 28(1942), pp. 535-537.

[2] B. Schweizer and A.Sklar, Probabilistic Metric Spaces, North-Holland Series in Probabilistic and Applied Mathematics, North-Holland, New York, NY, USA, (1983).

[3] A. N. Serstnev, On the motion of a random normed spaces, Doklady Akademii Nauk SSSR, vol. 149, no. 2(1963),280-283, English translation in Soviet Mathematics. Doklady , vol. 4(1963), 388390.

[4] B. Schweizer and A.Sklar, Statistical metric spaces, Pacific Journal of Mathematics vol. 10(1960), 313-334.

[5] S. M. Ulam, Problems in Modern Mathematics, Science Editions, John Wiley and Sons, (1964).

[6] D. H. Hyers, On the stability of the linear functional equation, Proc. Nat. Acad. Sci. U.S.A. 27 (1941), 222-224.

[7] D. H. Hyers, G. Isac, and Th. M. Rassias, Stability of Functional Equations in Several ariables , Birkhauser, Basel(1998).

[8] S. Czerwik, Functional Equations and Inequalities in Several Variables, World Scientific, River Edge, NJ, (2002).

[9] D. Deses, On the representation of non-Archimedean objects, Topology Appl. 153 (2005), no. 56, 774-785.

[10] W. Fechner, Stability of a functional inequality associated with the Jordan-von Neumann functional equation, Aequationes Math. 71 (2006), no. 1-2, 149-161.

[11] P. Gvavruta, A generalization of themHyers-Ulam-Rassias stability of approximately additive mappings, J. Math. Anal. Appl. 184 (1994), no. 3, 431-436. 
[12] K. Hensel, Ubereine news Begrundung der Theorie der algebraischen Zahlen, Jahresber. Deutsch. Math. Verein 6 (1897), 83-88.

[13] K. W. Jun and H. M. Kim, On the Hyers-Ulam-Rassias stability of a general cubic functional equation, Math. Inequal. Appl., 6 (1) (2003), 87-95.

[14] Y. S. Lee and S. Y. Chung, Stability of the Jensen type functional equation, Banach J. Math. Anal., 1 (1) (2007), 91-100.

[15] K. Mengar, Statistical metrics, Proc. Nat. Acad. Sci., 28 (1942), 535-537.

[16] Th. M. Rassias, On the stability of the linear mapping in Banach spaces, Proc. Amer. Math. Soc., 72 (1978), 297-300.

[17] Th. M. Rassias, On the stability of functional equations and a problem of Ulam, Acta Appl. Math., 62 (2000), 123-130.

[18] S. M. Ulam, Problems in Modern Mathematics, Science ed., John Wiley and Sons, New York, (1960).

[19] A. N. Serstnev, On the notion of a random normed space, Dokl. Akad. Nauk.149 (1963), 280283.

[20] E. Movahednia, Fuzzy Stability of Quadratic Functional Equations in General Cases, ISRN Mathematical Analysis, Hindawi, (2011).

[21] E. Movahednia, S. Eshtehar, Y. Son, Stability of Quadratic Functional Equations in Fuzzy Normed Spaces, Int. Journal of Math. Analysis, Vol. 6, no. 48, (2012), 2405 - 2412

[22] D. Mihet, V. Radu, Generalized pseudo-metrics and fixed points in probabilistic metric spaces, Carpathian Journal of Mathematics, 23, 1-2 (2007), 126-132. 